\title{
Eco-Biological Assessment of Main Forage Grain Crop and Legumes in Pastures Hayland of Shirvan Territory
}

\author{
T. E. Gasimzade \\ Department of Agrarian Sciences of ANAS, Baku, Azerbaijan \\ E-mail: nushana_kasimzade@yahoo.com
}

Received: May 16, 2015 Accepted: June 5, 2015

doi:10.5296/jbls.v6i2.7925 URL: http://dx.doi.org/10.5296/jbls.v6i2.7925

\begin{abstract}
Eco-biological properties of botanical teams of fodder grains and legumes which is highly efficient in terms distributed in Shirvan zone of Azerbaijan have been studied. During investigations 76 species from Cereal family, and 45 species from legumes were defined. Some of them are as common in the area where others been determined. It was known during biotopological analysis of investigated species that 20-25 species of legumes are oommon in bushes, 18 species in forest, forest edge, arid forest biotops, grape fields, and gardens, and 4 species in stoned cliffs. Variation of these species on zones is non-equal. 47 species were found in lowland mountain zone, 17 species in middle mountain zone and 23 species are common in upland mountain zone. Analysis of ecological groups of cereals showed that 10 species grow in mesophyte, 50 species in xerophyte, 16 species in mesoxerophyte condition. 3 species of Legumes grow in mesophyte condition, 24 species in xerophyte, 19 species in mesoxerophyte condition.
\end{abstract}

Keywords: Grain, Legume, Forage crops, Meadowsç Pastures, Shirvan (Azerbaijan), Ecological assessment

\section{Introduction}

Geo-biomorphological investigation in Azerbaijan has been started since the 50th of last century. Geographers M.Musaibov, botanists acad. V.Hajiyev [Gadzhiyev 1983; Hajiyev 1990], soilscientists acad. M.Salayev [Salayev 1991] and acad. G.Mammadov [Mammadov, et all 2006; Mammadov 2007] played an important role in implementing the investigations.

Geological structure (neotectonical) - as e result of neotectonical movements occur within Azerbaijan as differential movement's macrostructure, Kur-Arazdepressions were formed [Agaguliyev 2000; Aliyev, et all 1976]. 


\section{$\triangle 1$ Macrothink}

Investigated Area covers Gobustan, Goychay, Hajigabul, Kurdemir, Agsu, Ismayilli and Shamakhi (partly) and bordered with Neogen upland, Langebiz mountains from north, from north-east Small

Harami range, from south Kura river, south east Hajigabul river and from west part of Girdmanchai river. Some parts of the area are $11 \mathrm{~m}$ less than ocean level (Javad village), are equal to ocean level (Hajigabul, Kurdemir) whereas some parts are higher than ocean level up to 50 meters (Agsu city). Length of investigation area on linear transect is $80 \mathrm{~km}$ and width is approximately $40 \mathrm{~km}$, general area is $3200 \mathrm{~km}$ square. Geographical coordinates of the territory: 40-41 30 north latitue 488-49 longtitu.

It is dedicated to study grain crops and legume that is base of forage crop in the territory. Bioecological properties of grain crops and legumes that spread above mentioned zones.

\section{Material and Methods}

Morphological, systematical, ecological -geographical, phenological and other methods were used in determination of plants. During analysis of flora it was based on C. Raunker [Raunkiaer 1934] and Brown-Blanke [Braun-Blanguet 1964], I.Q. Serebryakov [Serebryakov 1964] about life form, geographical and areal types of A.A. Grosshame [Grossgeim 1936], N.N. Portenier [Portenier 2000; Portenier 2000], ecological groups A.R. Shennikova [Shennikov 1938]. In the list of flora there were reflected information about each specie's family, its subfamily as well as their life form, ecological groups, geographical and areal types, flowering and harvest periods, endemism and so on. Names of toxonoms were given according to S.K. Chereranov [Cherepanov 1995].

\section{Discussion}

In order to learn Shirvan vegetation and ecology, geobotanical investigation were carried out on the following routes in 2008-2014. Investigated areas were studied in different seasons by route methods. Investigations were carried out in next districts (table 1).

Table 1. Investigations were carried out in next districts of Azerbaijan

\begin{tabular}{|l|l|}
\hline $\begin{array}{l}\text { Date } \\
\text { investigations }\end{array}$ & Place of investigations \\
\hline 06.06.08. & Goychay district, Mirzehusainli village meadow legume-grain grass \\
\hline 06.06 .08$. & $\begin{array}{l}\text { Goychay district Qarayazi village (near cemetery) wormseed - legume in semi-desert } \\
\text { plants type }\end{array}$ \\
\hline 06.06 .08$. & Agsu district,Purhasanli village, grat-brown soils, barley-thistle plants \\
\hline 07.06 .08$. & $\begin{array}{l}\text { Agsu district, Nuydu munipalicy, brown mountain-forest soils under forest vegetation on } \\
\text { third tier grain -legume-different grasses were gathered }\end{array}$ \\
\hline $17.06 .08-19.06 .08$. & $\begin{array}{l}\text { Ismailly district, Talistan village mountain-brown soils near forest from third tier } \\
\text { grains-different grasses were gathered }\end{array}$ \\
\hline $17.06 .08-19.06 .08$. & $\begin{array}{l}\text { Ismailly, Kurdmashi village, planting areas, plain relief, from dark gray-brown soils } \\
\text { inmeadows mainly legumes and grain crops were garhered }\end{array}$ \\
\hline 07.05 .08$. & Hajigabul district, Small harami range semi desert vegatation \\
\hline
\end{tabular}




\begin{tabular}{|c|c|}
\hline 08.06.08. & $\begin{array}{l}\text { from Gobustan district, Gurbanchi village less inclinated plain, gray brown soil, in semi } \\
\text { desert vegatation ephemere formassion herbaries were gathered }\end{array}$ \\
\hline 03.11.08. & $\begin{array}{l}\text { from Gobustan district, Gurbanchi less inclinated plain, gray brown soil, in semi desert } \\
\text { vegatation wormwood -ephemere formassion herbaries were gathered and had taken } \\
\text { photos }\end{array}$ \\
\hline 09.06.09-10.06.09. & $\begin{array}{l}\text { water plants that spread in Hajigabul district, Shahriyar village (Kur bank), Tugay forest } \\
\text { vegetation were investigated and herbary materials were gathered and photos had been } \\
\text { taken }\end{array}$ \\
\hline 20.07.10-23.07.10. & $\begin{array}{l}\text { water plants that spread In Hajigabul district, Hajigabul lake and upland Shirvan } \\
\text { collector's banks were investigated and their species composition was defined }\end{array}$ \\
\hline 20.10.11.-24.10.11. & $\begin{array}{l}\text { Desert vegetation (saltwort) near Mugan, Qarasu, Padar villages of Hajigabul district was } \\
\text { geobotanically statied }\end{array}$ \\
\hline 12.10.12.-13.10.12. & $\begin{array}{l}\text { Plants near Pirili, Jarli, Qocali in Kurdemir District, Arabushagi, Kendoba in Agsu } \\
\text { district were investigated geobotanically and herbari materials were collected }\end{array}$ \\
\hline 17.06.12. & 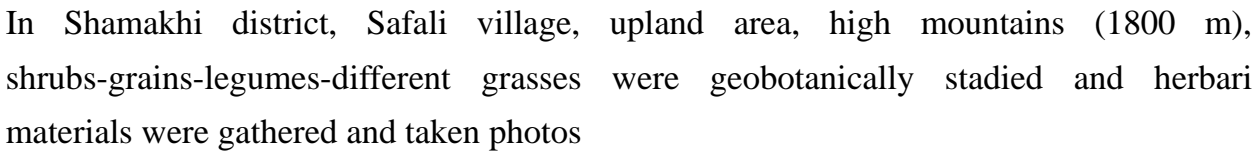 \\
\hline 17.06.12. & $\begin{array}{l}\text { In Shamakhi district, Melhem village, mountain - brown, near forest meadow vegetation } \\
\text { were observed and herbari materials were collected and taken photos }\end{array}$ \\
\hline 22.05.13.-24.05.13. & $\begin{array}{l}\text { In Hajigabul near Talish village and Tugay forest vegetation near Kur River bank was } \\
\text { observed and herbari materials were collected and photos taken }\end{array}$ \\
\hline 16.05.13.-20.05.13. & $\begin{array}{l}\text { water plants in Hajigabul district, near Hajigabul lake,Shirvan Collector, Kur river banks } \\
\text { were investigated and herbari materials were collected }\end{array}$ \\
\hline 7.10.13.-10.10.13. & $\begin{array}{l}\text { semidersert vegetation (white wormwood ) in Hajigabul district, Small Harami range was } \\
\text { statied and herbari materials were collected and photos taken }\end{array}$ \\
\hline 17.10.13-19.10.13. & $\begin{array}{l}\text { geobotanical investigation of desert vegetation of Hajigabul district in Mugan, Qarasu, } \\
\text { villages and Karrar village in Kurdemir district was carried out and herbari materials } \\
\text { were collected and photos taken }\end{array}$ \\
\hline 3.11.14.-6.11.14. & $\begin{array}{l}\text { desert vegetation near Qarasu and Padar villages in Hajigabul was investigated, herbari } \\
\text { materials were colleced and photos were taken }\end{array}$ \\
\hline 20.05.14.-22.05.14. & $\begin{array}{l}\text { gobotanical investigation of plants near Qaraqoyunlu, Arabushagi, rehimli, Kendoba } \\
\text { villages in Agsu was carried out and herbari materials were collected and photos of } \\
\text { characteric areas were taken }\end{array}$ \\
\hline 10.10.14.-12.10.14. & $\begin{array}{l}\text { geobotanical investigation of vegetation near Karrar, Sigirri and Mollakend villages in } \\
\text { Kurdemir disrtict was carried out and herbari materials were collected }\end{array}$ \\
\hline
\end{tabular}

Scarce and agricultural important fodd and forage plants' 17 seed samples that cover 17 species and 10 types were collected, their descriptors were defined and given to Genebank in order to sustainable usage and restoration as genetical materials.

\section{Experiments and Discussions}

During investigations 76 species from Cereal family, from legumes 45 species were defined. Some of them are common in the area where as some of them are scarce (figure. 1,2). 


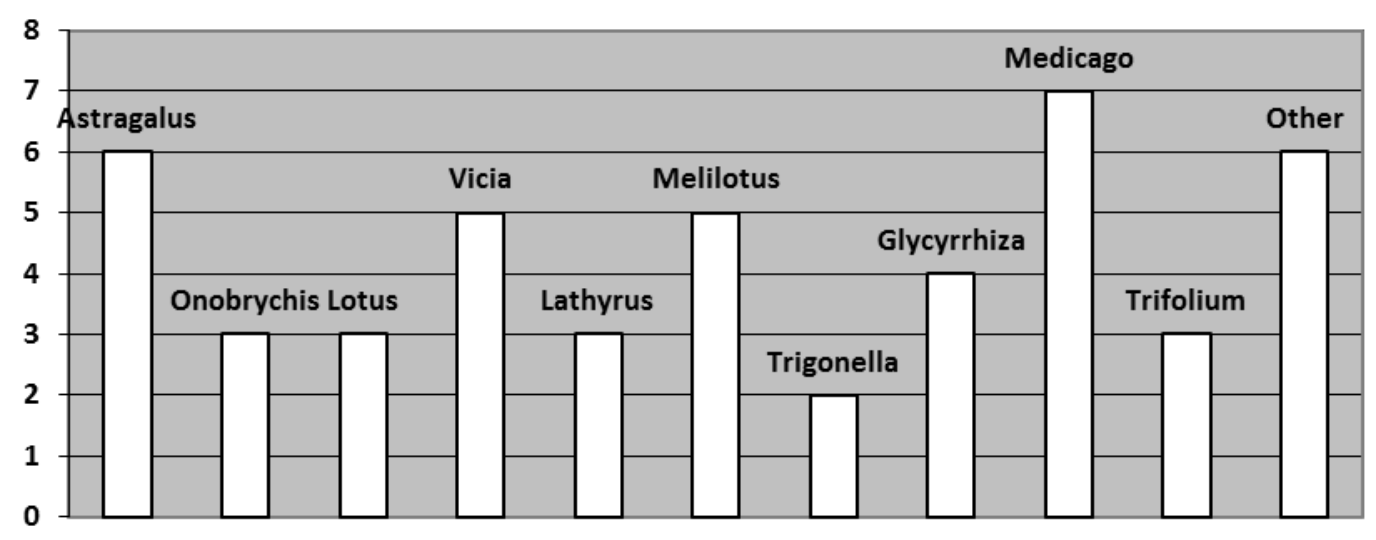

Figure 1. The number of types according to species of legumes

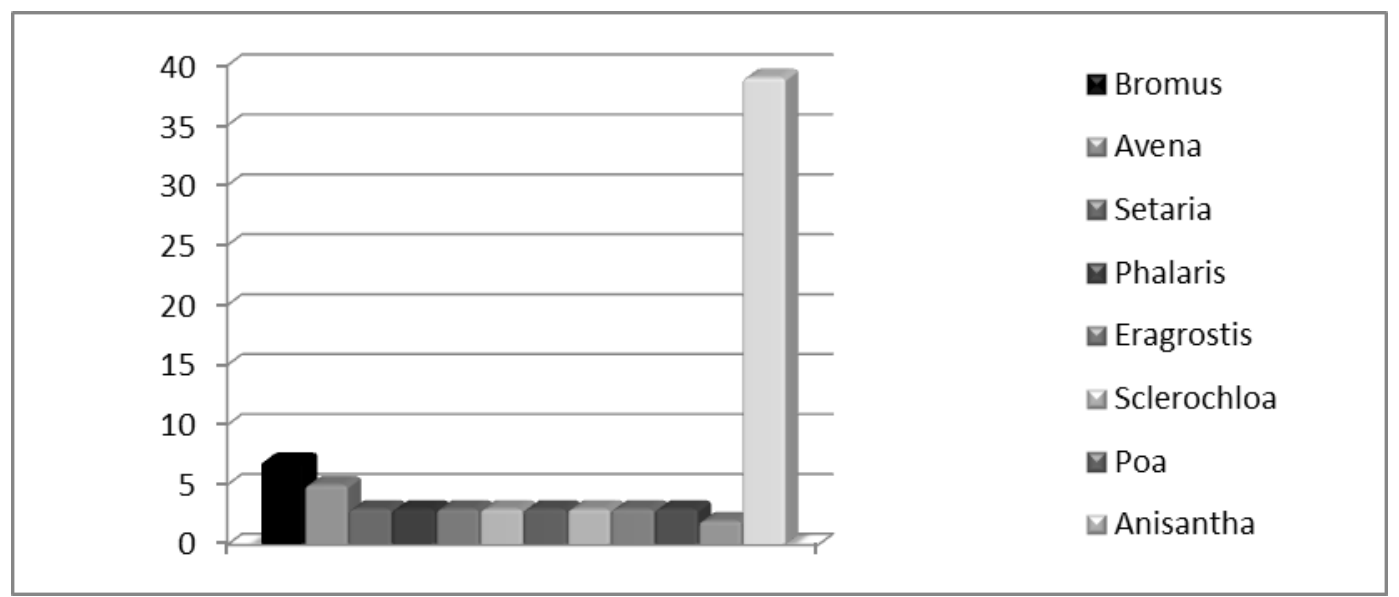

Figure 2. The number of types according to species of cereals

It is obvious from figures that in Shirvan area, Cereals such as Bromus L., Avena L., and Legumes such as Astragalus and Meticago types are common.

During investigation, ecological analysis, analysis about biotops where investigated species spread and their differensationaccording to zones and ecological groups (according to water) and other ecological issues were studies as well as toxsonomical analysis. This investigation was carried out according to literature information and was changed during expeditiond to the territory. It was known during biotopological analysis of investigated species that 20-25 species of legumes are oommon in bushes, 18 species in forest, forest edge, arid forest biotops, grape fields, gardens, 4 species in stoned cliffs. Variantion of these species on zones is non-equal. In lowland mountain zone 47 species, 17 species middle mountain zone and in upland mountain zone 23 species are common. 


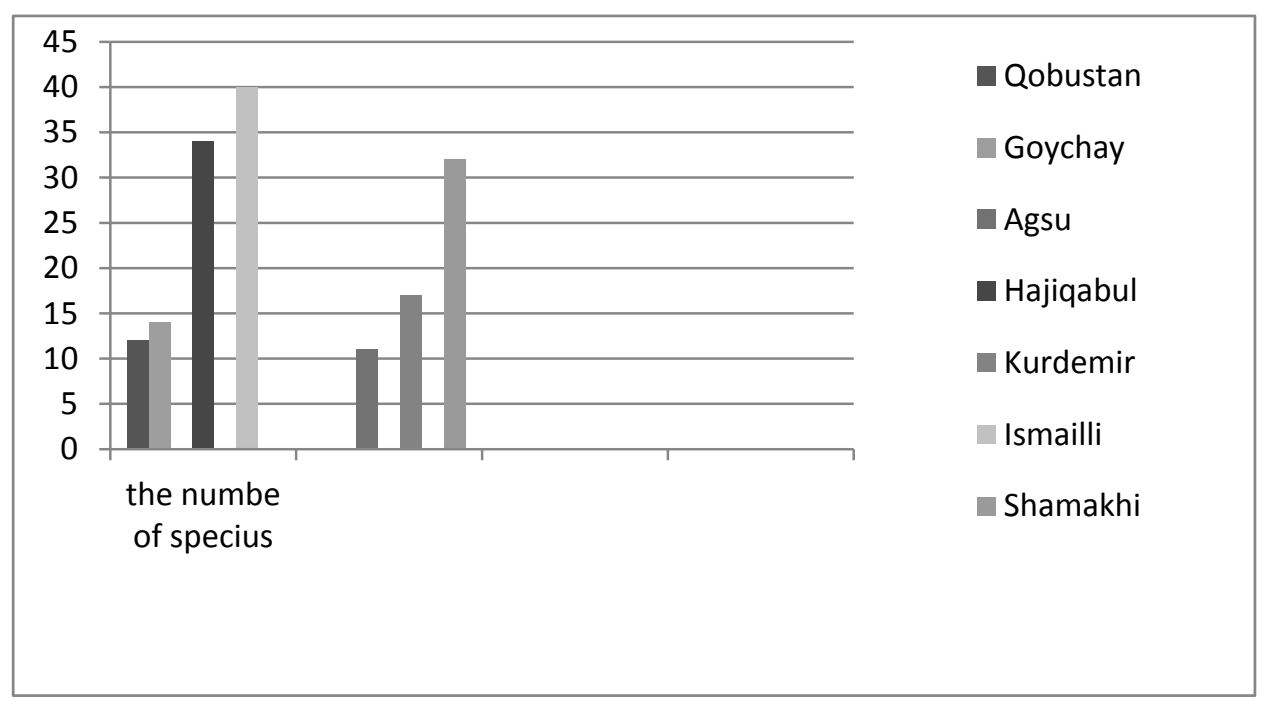

Figure 3. Distribution of cereals family according to districts

Investigations and collected herbari materials how that distribution of legume family according to districts are the following (figure 4.)

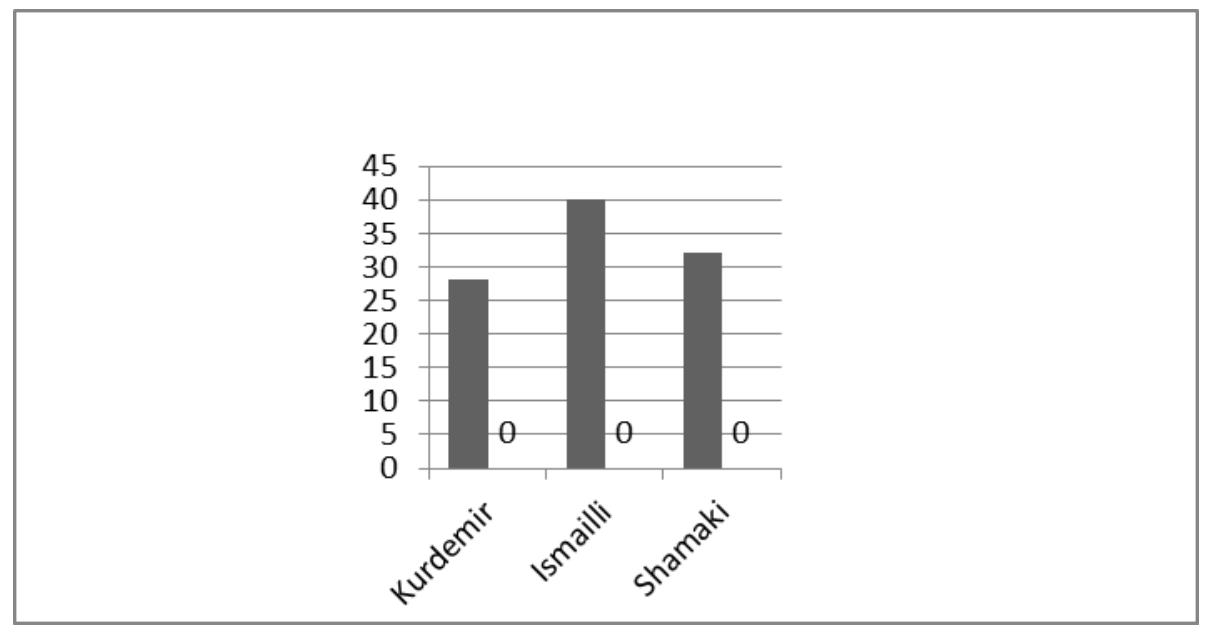

Figure 4. Distribution of Legume family according to districts

Monitorings carried out during expedition showed that, physical-geogaphical and anthropogenic factors play an important role in ecological groups of species and distribution on mountain zones. biotopological, ecological, geographical analysis of studied species are illustrated in figures $(5,6)$ and shown in Table 2 and Table 3. 


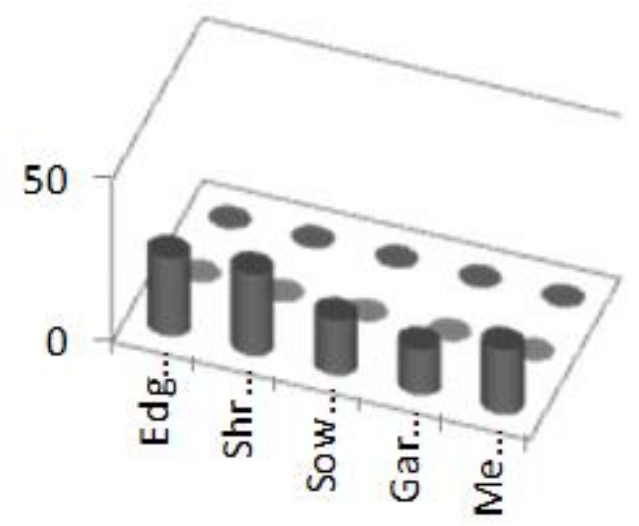

Figure 5. Distrubition of Cereal family species on important biotops

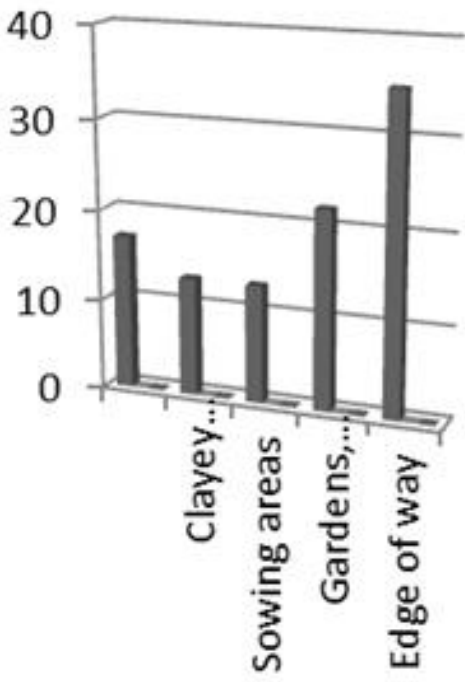

Figure 6. Distrubition of Cereal family species on important biotops

Table 2. Eco-biological properties of individuals from Cereals family in Shirvan territory

\begin{tabular}{|l|l|c|l|l|c|}
\hline $\begin{array}{l}\text { Names of families, types } \\
\text { and species }\end{array}$ & $\begin{array}{l}\text { Life forms by } \\
\text { Serebryakov and } \\
\text { Raunkier }\end{array}$ & Geographical types & Areal types & $\begin{array}{l}\text { Ecological } \\
\text { groups }\end{array}$ & $\begin{array}{c}\text { Phenological } \\
\text { phase }\end{array}$ \\
\hline \multicolumn{5}{|c|}{ Poaceae Barnhart } \\
\hline $\begin{array}{l}\text { 1.Imperata cylindrica } \text { (L.) } \\
\text { Raeusch. }\end{array}$ & perennial. HK & $\begin{array}{c}\text { Mediterranean-Iran - } \\
\text { Turan }\end{array}$ & Ancient.Ar.d. & Mesophyte & V-VII \\
\hline $\begin{array}{l}\text { 2. Tragus racemosus } \text { (L.) } \\
\text { All. }\end{array}$ & annual. T. & Mediterranen - Ir. & Ancient.Ar.d. & Xerophyte & VI- VIII \\
\hline $\begin{array}{l}\text { 3. Paspalum paspalodes } \\
\text { (Michx.) Scribn. }\end{array}$ & perennial. HK & Adventiv & Adventiv & Mesophyte & VI-IX (XI) \\
\hline 4. P. dilatatum Poir. & perennial. HK & Adventiv & Adventiv & Xerophyte & VII - VIII \\
\hline
\end{tabular}




\begin{tabular}{|c|c|c|c|c|c|}
\hline $\begin{array}{l}\text { 5. Eriochloa succincta } \\
\text { (Trin.) Kunth }\end{array}$ & annual. $\mathrm{T}$. & Turan & Səhra & Hygrophyte & (2/ VIII) IX-XI \\
\hline $\begin{array}{l}\text { 6. Digitaria violascens } \\
\text { Link }\end{array}$ & annual. $\mathrm{T}$. & Adventiv & Adventiv & Xerophyte & VII - IX \\
\hline 7. D. sanguinalis (L.) Scop. & annual. T. & Boreo. - trop. & Adventiv & MezoXerophyte & VII - X \\
\hline $\begin{array}{l}\text { 8. Echinochloa crusgalli } \\
\text { (L.) Beauv. }\end{array}$ & annual. T. & Boreo. -trop. & Adventiv & MezoXerophyte & VII - IX \\
\hline $\begin{array}{l}\text { 9. Setaria verticillata (L.) } \\
\text { Beauv. }\end{array}$ & annual. $\mathrm{T}$. & Palearkt. -subtrop. & Adventiv & Xerophyte & VI-IX \\
\hline 10. S. pumila (Poir.) Schult. & annual. T. & Trop. - subtrop. & Adventiv & Xerophyte & VI-X \\
\hline 11. S.viridis (L.) Beauv. & annual. T . & Palearkt. & Boreal & Mesophyte & (V)VI-VIII(X) \\
\hline 12. Phalaris minor Retz & annual. T. & Medit. -Ir. - Sind & Ancient.Ar.d. & Xerophyte & IV-V, VI \\
\hline 13. Ph. brachystachys Link & annual. T. & Mediterranen & Ancient.Ar.d. & Xerophyte & V-VI, VI - VII \\
\hline 14. Ph. paradoxa L. & annual. T & Mediterranen & Ancient.Ar.d. & Xerophyte & V, VI-VII \\
\hline $\begin{array}{l}\text { 15.Phalaroides } \\
\text { arundinacea (L.) Rauschert }\end{array}$ & perennial. $\mathrm{K}$ & Holarkt. & Boreal & Hygrophyte & VI-VII, VII -IX \\
\hline $\begin{array}{l}\text { 16. Stipa prilipkoana } \\
\text { Grossh. }\end{array}$ & perennial. HK & Iran & Ancient.Ar.d. & Xerophyte & $\mathrm{V}$ \\
\hline 17. S. caspia C.Koch & perennial. HK & Iran - Turan & Ancient.Ar.d. & Xerophyte & $\mathrm{V}-\mathrm{VI}$ \\
\hline $\begin{array}{l}\text { 18. Crypsis schoenoides } \\
\text { (L.) Lam. }\end{array}$ & annual. T & Mediter. - Tur. & Ancient.Ar.d. & Hygrophyte & (VI)VII- X (XI) \\
\hline $\begin{array}{l}\text { 19. Phleum paniculatum } \\
\text { Huds. }\end{array}$ & annual. $\mathrm{T}$ & Mediter. -Tur. & Ancient.Ar.d. & MezoXerophyte & V-VI (VII) \\
\hline $\begin{array}{l}\text { 20. Alopecurus } \\
\text { arundinaceus Poir. }\end{array}$ & perennial. HK & Palearkt. & Boreal & MezoXerophyte & V-VII \\
\hline 21. A. myosuroides Huds. & annual. $\mathrm{T}$ & Mediter. -Ir. -Tur. & Ancient.Ar.d. & Mesophyte & $\mathrm{V}-\mathrm{VI}$ \\
\hline $\begin{array}{l}\text { 22. Polypogon } \\
\text { monspeliensis (L.) Desf. }\end{array}$ & annual. $\mathrm{T}$ & Mediter. -Ir. -Tur. & Ancient.Ar.d. & Hygrophyte & V-VII \\
\hline 23. Agrostis gigantea Roth & perennial. HK & Holarkt. & Boreal & Mesophyte & VI-VII \\
\hline $\begin{array}{l}\text { 24. Calamagrostis epigeios } \\
\text { (L.) Roth }\end{array}$ & perennial. $\mathrm{K}$ & Palearkt. & Boreal & Hygrophyte & VI-VIII \\
\hline 25. Avena clauda Durieu & annual. T & Mediter. & Ancient.Ar.d. & Xerophyte & $\mathrm{V}$ \\
\hline 26. A. eriantha Durieu & annual.T & Mediter. & Ancient.Ar.d. & Xerophyte & 2/IV-V \\
\hline 27. A. fatua $\mathrm{L}$. & annual. T & Western palearkt. & Boreal & Xerophyte & VI-VII \\
\hline 28. A. persica Steud. & annual. T & Tur. & Səhra & Xerophyte & IV-VI (VII) \\
\hline 29. A. trichophylla C. Koch & annual. T & Front Asia & Ancient.Ar.d. & Xerophyte & 2/IV-V/VI \\
\hline $\begin{array}{l}\text { 30. Cynodon dactylon (L.) } \\
\text { Pers. }\end{array}$ & perennial. HK & Mediter.-Ir. Tur. & Ancient.Ar.d. & MezoXerophyte & VI-IX \\
\hline 31.Arundo donax L. & perennial. HK & Mediter. & Ancient.Ar.d. & MezoXerophyte & VIII-X \\
\hline $\begin{array}{l}\text { 32. Phragmites australis } \\
\text { (Cav.) Trin. ex Steud. }\end{array}$ & perennial. $\mathrm{K}$ & Holarkt. & Boreal & Hidrofit & VII-X \\
\hline
\end{tabular}




\begin{tabular}{|c|c|c|c|c|c|}
\hline $\begin{array}{l}\text { 33. Eragrostis pilosa } \\
\text { (L.)Beauv. }\end{array}$ & annual. T & Boreo-subtrop. & Adventiv & MezoXerophyte & VII- VIII (X) \\
\hline 34.E.minor Host & annual. T & Mediter..- Ir.-Tur. & Ancient.Ar.d. & MezoXerophyte & VI-IX \\
\hline 35. E. starosselskyi Grossh. & perennial. HK & Ir.-Tur. & Ancient.Ar.d. & Xerophyte & 2/VI-VII (X) \\
\hline $\begin{array}{l}\text { 36. Rostraria glabriflora } \\
\text { (Trautv.) Czer. }\end{array}$ & annual. $\mathrm{T}$ & Mediter.- Ir.-Tur. & Ancient.Ar.d. & MezoXerophyte & IV-VI \\
\hline $\begin{array}{l}\text { 37. Catabrosa aquatica } \\
\text { (L.)Beauv. }\end{array}$ & perennial. $\mathrm{K}$ & Holarkt. & Boreal & Hygrophyte & V-VII \\
\hline $\begin{array}{l}\text { 38.Aeluropus littoralis } \\
\text { (Gouan) Parl. }\end{array}$ & perennial. HK & Mediter.-Ir. & Ancient.Ar.d. & MezoXerophyte & V-VII \\
\hline 39.Cynosurus echinatus L. & annual. T & Mediter. & Ancient.Ar.d. & Xerophyte & V-VII \\
\hline $\begin{array}{l}\text { 40.Sclerochloa dura (L.) } \\
\text { Beauv. }\end{array}$ & annual. $\mathrm{T}$ & Mediter. -Tur. & Ancient.Ar.d. & Xerophyte & IV-VII \\
\hline $\begin{array}{l}\text { 41. Schismus arabicus Nees } \\
\text { Thell. }\end{array}$ & annual. T & $\begin{array}{l}\text { Eastern- Mediter. } \\
\text {-Ir.-Tur. }\end{array}$ & Ancient.Ar.d. & Xerophyte & IV-VI \\
\hline 42.S. barbatus (L.) Thell. & annual. $\mathrm{T}$ & Mediter. & Ancient.Ar.d. & Xerophyte & IV-VI \\
\hline 43. Poa bulbosa L. & perennial. HK & Mediter.-Sarm. & Ancient.Ar.d. & Xerophyte & III -IV,IV \\
\hline 44.P. annua L. & annual. $\mathrm{T}$ & Kosmopolit & Kosmopolit & Mesophyte & V-VII, VI -IX \\
\hline 45. P. trivialis L. & perennial. HK & Palearktic & Boreal & Mesophyte & $\begin{array}{c}\text { VI-VII, VII - } \\
\text { VIII }\end{array}$ \\
\hline $\begin{array}{l}\text { 46. Catabrosella humilis } \\
\text { (Bieb.)Tzvel. }\end{array}$ & perennial. HK & Sarmart & Grey & Xerophyte & IV-V \\
\hline $\begin{array}{l}\text { 47. Puccinellia gigantea } \\
\text { (Crossh.) Grossh. }\end{array}$ & perennial. HK & Turan & Desert & Mesophyte & $\mathrm{V}$ \\
\hline $\begin{array}{l}\text { 48.P. bulbosa (Grossh.) } \\
\text { Grossh. }\end{array}$ & perennial. HK & Eastern Transcaucasia & Desert & Mesophyte & $\mathrm{V}$ \\
\hline 49. Festuca pratensis Huds. & perennial. HK & Europe & Boreal & Xerophyte & V- VIII \\
\hline 50. F. arundinacea Schreb. & perennial. HK & Western -palearkt. & Boreal & Mesophyte & V-VIII \\
\hline $\begin{array}{l}\text { 51. Vulpia myuros } \\
\text { (L.)C.C.Gmel. }\end{array}$ & annual. $\mathrm{T}$ & Western & Ancient.Ar.d. & MezoXerophyte & IV-VI \\
\hline $\begin{array}{l}\text { 52. Parapholis incurva (L.) } \\
\text { C.E. Hubb. }\end{array}$ & annual. $\mathrm{T}$ & Mediter.-Ir.-Tur. & Ancient.Ar.d. & Xerophyte & IV-VI \\
\hline $\begin{array}{l}\text { 53. Pholiurus pannonicus } \\
\text { (Host) Trin. }\end{array}$ & annual. $\mathrm{T}$ & Pan.- Sarmat & Grey & Xerophyte & V- VI \\
\hline $\begin{array}{l}\text { 54.Psilurus incurvus } \\
\text { (Gouan) Schinz. \&Thell. }\end{array}$ & anual. T & Western & Ancient.Ar.d. & MezoXerophyte & IV-V \\
\hline $\begin{array}{l}\text { 55. Scleropoa rigida (L.) } \\
\text { Griseb. }\end{array}$ & annual. $\mathrm{T}$ & Mediter. & Ancient.Ar.d. & Xerophyte & $\mathrm{V}$ \\
\hline $\begin{array}{l}\text { 56. Anisantha sterilis (L.) } \\
\text { Nevski }\end{array}$ & annual. $\mathrm{T}$ & Mediter.- Tur. & Ancient.Ar.d. & Xerophyte & IV-VI \\
\hline 57. A. rubens (L.) Nevski & annual. $\mathrm{T}$ & Mediter. & Ancient.Ar.d. & Xerophyte & III- VI \\
\hline 58. A. tectorum (L.) Nevski & annual. T & Mediter.-Ir.-Tur. & Ancient.Ar.d. & Xerophyte & (III) IV - VI \\
\hline
\end{tabular}




\begin{tabular}{|c|c|c|c|c|c|}
\hline 59. Bromus arvensis L. & biennial. HK & Palearkt. & Boreal & Xerophyte & V-VII \\
\hline 60.B. squarrosus L. & biennial. HK & Mediter. & Ancient.Ar.d. & Xerophyte & VI-VII \\
\hline 61. B. japonicus Thunb. & annual. $\mathrm{T}$ & Europe - Mediter. & Boreal & MezoXerophyte & V-VII \\
\hline 62.B. commutatus Schrad. & annual. $\mathrm{T}$ & Europe & Boreal & Xerophyte & V- VII \\
\hline 63. B. mollis L. & annual. $\mathrm{T}$ & Europe & Boreal & Xerophyte & V-VII \\
\hline 64. B. scoparius L. & annual. T & Mediter.-Ir.-Tur. & Ancient.Ar.d. & MezoXerophyte & IV- VII \\
\hline 65. B. danthoniae Trin. & annual. $\mathrm{T}$ & Ir.-Tur. & Ancient.Ar.d. & Xerophyte & V-VII \\
\hline $\begin{array}{l}\text { 66.Trachynia distachya (L.) } \\
\text { Link }\end{array}$ & annual. $\mathrm{T}$ & Mediter.-Ir.-Tur. & Ancient.Ar.d. & Xerophyte & IV-VI, VI - VII \\
\hline 67. Lolium rigidum Gaudin & annualr. $\mathrm{T}$ & Mediter.-Ir. & Ancient.Ar.d. & Xerophyte & $\mathrm{V}-\mathrm{VI}$ \\
\hline $\begin{array}{l}\text { 68. Elytrigia repens (L.) } \\
\text { Nevski }\end{array}$ & perennial. HK & Palearkt. & Boreal & Mesophyte & $\mathrm{VI}-\mathrm{VIII}$ \\
\hline $\begin{array}{l}\text { 69. Eremopyrum triticeum } \\
\text { (Gaertn.) Nevski }\end{array}$ & annual. T & Sarmat & Grey & Xerophyte & IV-V (1/ VI) \\
\hline $\begin{array}{l}\text { 70. E. orientale (L.) } \\
\text { Jaub.\&.Spach }\end{array}$ & annual. T & Mediter.-Ir.- Tur. & Ancient.Ar.d. & Xerophyte & IV-VI \\
\hline $\begin{array}{l}\text { 71. E. distans (C.Koch) } \\
\text { Nevski }\end{array}$ & annual. $\mathrm{T}$ & Ir.-Tur. & Ancient.Ar.d. & Xerophyte & $\mathrm{V}-1 / \mathrm{VI}$ \\
\hline $\begin{array}{l}\text { 72. Aegilops cylindrica } \\
\text { Host }\end{array}$ & annual. T & $\begin{array}{l}\text { The east - } \\
\text { Mediter.-Ir.-Tur. }\end{array}$ & Ancient.Ar.d. & Xerophyte & $2 / \mathrm{V}-\mathrm{VI}$ \\
\hline 73. Ae. triuncialis L. & annual. T & Mediter.-Ir.-Tur. & Ancient.Ar.d. & Xerophyte & $\mathrm{V}-\mathrm{VI}$ \\
\hline 74. Hordeum bulbosum L. & perennial. HK & Mediter. & Ancient.Ar.d. & MezoXerophyte & V- 1/VII \\
\hline 75. H. leporinum Link & annual. $\mathrm{T}$ & Mediter.- Ir. - Tur. & Ancient.Ar.d. & Xerophyte & 2/IV-VI( 1/ VII) \\
\hline 76. H. geniculatum All. & annual. T & Mediter. & Ancient.Ar.d. & MezoXerophyte & $\mathrm{V}-\mathrm{VI}$ \\
\hline
\end{tabular}

Table 3. Eco-biological properties of individuals from legumes family in Shirvan territory

\begin{tabular}{|c|c|c|c|c|c|}
\hline $\begin{array}{c}\text { Names of families, types and } \\
\text { species }\end{array}$ & $\begin{array}{c}\text { Life forms by } \\
\text { Serebryakov and } \\
\text { Raunkier }\end{array}$ & $\begin{array}{l}\text { Geographical } \\
\text { types }\end{array}$ & Areal types & $\begin{array}{l}\text { Ecological } \\
\text { groups }\end{array}$ & $\begin{array}{c}\text { Phenological } \\
\text { phase }\end{array}$ \\
\hline \multicolumn{6}{|c|}{ Fabaceae Lindl. } \\
\hline $\begin{array}{l}\text { 1.Lagonychiumfarctum } \\
\text { (Banks\&Soland.) Bobr. }\end{array}$ & $\begin{array}{c}\text { Stony, rocky places, } \\
\text { Ch }\end{array}$ & $\begin{array}{l}\text { The east } \\
\text { Mediter.-Ir.-Tur. }\end{array}$ & Ancient.Ar.d. & MezoXerophyte & V-VI, VII-IX \\
\hline 2. Trigonella calliceras Fisch. & annual. T & Hirk. & Ancient & Xerophyte & IV-V, V-VI \\
\hline 3. T. monspeliaca L. & annual. $\mathrm{T}$ & Mediter. & Ancient.Ar.d. & Xerophyte & IV-V. V-VI \\
\hline $\begin{array}{l}\text { 4. Medicago caerulea Less. ex } \\
\text { Ledeb. }\end{array}$ & perennial. HK & Sarm. & Grey & Xerophyte & V-(VIII), VIII-IX \\
\hline 5. M.lupulina L. & Iki.HK & Palearkt. & Boreal & MezoXerophyte & IV-VII \\
\hline 6. M. orbicularis (L.) Bartalini & annual. T & Mediter. & Ancient.Ar.d. & MezoXerophyte & IV-VII \\
\hline 7. M. truncatula Gaertn. & annual. $\mathrm{T}$ & Mediter. & Ancient.Ar.d. & Xerophyte & IV-VII \\
\hline 8. M. rigidula (L.) All. & annual. $\mathrm{T}$ & Mediter. & Ancient.Ar.d. & Xerophyte & IV-VI \\
\hline 9. M. minima (L.) Bartalini & annualT & $\begin{array}{l}\text { Mediter.- Front } \\
\text { Asia }\end{array}$ & Ancient.Ar.d. & Xerophyte & IV-V \\
\hline
\end{tabular}




\begin{tabular}{|c|c|c|c|c|c|}
\hline 10. M. denticulata Willd. & annual. $\mathrm{T}$ & Mediter. & Ancient.Ar.d. & MezoXerophyte & IV-VI(VII) \\
\hline $\begin{array}{l}\text { 11. Melilotus officinalis (L.) } \\
\text { Pall. }\end{array}$ & biennial. HK & Western -palearkt. & Boreal & MezoXerophyte & V-VI, VI-IX \\
\hline 12. M. neapolitanus Ten. & annual. $\mathrm{T}$ & Mediter. & Ancient.Ar.d. & Xerophyte & $\mathrm{V}, \mathrm{VI}$ \\
\hline 13. M. indicus (L.) All. & annual. $\mathrm{T}$ & Mediter.-Ir.Tur. & Ancient.Ar.d. & MezoXerophyte & VI, V-VI \\
\hline 14. Trifolium arvense L. & annual. $\mathrm{T}$ & Western -palearkt. & Boreal & Xerophyte & VI-VII,VI-VIII \\
\hline 15. T. pratense L. & perennial. HK & Western -palearkt. & Boreal & MezoXerophyte & V-VII \\
\hline 16. T. lappaceum L. & annual. $\mathrm{T}$ & Mediter. & Ancient.Ar.d. & Xerophyte & V,VI \\
\hline $\begin{array}{l}\text { 17. Amoria bonannii (C. } \\
\text { Presl) Roskov }\end{array}$ & perennial. HK & Mediter. & Ancient.Ar.d. & Mesophyte & V-VI \\
\hline 18. A. repens (L.) C. Presl & perennial. HK & Palearkt. & Boreal & Mesophytet & V-VII, VII-VIII \\
\hline 19. A. resupinata (L.) Roskov & annual. T & Mediter. & Ancient.Ar.d. & MezoXerophyte & IV-V, V-VI \\
\hline $\begin{array}{l}\text { 20. Chrysaspis campestris } \\
\text { (Schreb.) Desv. }\end{array}$ & annual. $\mathrm{T}$ & Europa & Boreal & MezoXerophyte & V-VI, VI-VII \\
\hline $\begin{array}{l}\text { 21. Ch. micrantha (Viv.) } \\
\text { Hendrych }\end{array}$ & annual. $\mathrm{T}$ & Atl.- Mediter. & Boreal & MezoXerophyte & V-VI \\
\hline $\begin{array}{l}\text { 22. Astragalus asteriasStev. } \\
\text { ex Ledeb. }\end{array}$ & annual. $\mathrm{T}$ & Mediter. & Ancient.Ar.d. & Xerophyte & IV \\
\hline 23. A.hamosus L. & annual. $\mathrm{T}$ & South -Ir.Tur. & Ancient.Ar.d. & Xerophyte & IV-V, V-VI \\
\hline 24. o A. stevenianus DC. & perennial. HK & Iber. & Caucaus & Xerophyte & IV-V, VI \\
\hline 25. A. striatellus Pall. ex Bieb. & annual. $\mathrm{T}$ & Turan & Desert & Xerophyte & IV-V, V-VI \\
\hline 26. A. tribuloides Delile & annual. T & Mediter.- Ir.-Tur. & Ancient.Ar.d. & Xerophyte & III-IV \\
\hline 27. A. reticulatus Bieb. & annual. T & Sarmat & Grey & Xerophyte & V-VI \\
\hline 28. Glycyrrhiza echinata L. & perennial. HK & Unbeing known & M. o & Mesophyte & V-VII, VII-IX \\
\hline 29. G. aspera Pall. & perennial. HK & Turan & Desert & Xerophyte & (V)VII, VI (VII) \\
\hline 30. G. glabra L. & perennial. HK & Mediter.-Ir.-Tur. & Ancient.Ar.d. & MezoXerophyte & V-VII, VII-IX \\
\hline 31. G. foetidissima Tausch & perennial. HK & The east Europa & Boreal & MezoXerophyte & VI-VII, VII-VIII \\
\hline $\begin{array}{l}\text { 32. Coronilla scorpioides (L.) } \\
\text { Koch }\end{array}$ & annual. $\mathrm{T}$ & Mediter. & Ancient.Ar.d. & Xerophyte & IV-V \\
\hline $\begin{array}{l}\text { 33. Securigera varia (L.) } \\
\text { Lassen }\end{array}$ & perennial. HK & Europa & Boreal & MezoXerophyte & V-VII \\
\hline 34. Onobrychis cyri Grossh. & perennial. HK & $\begin{array}{l}\text { The north } \\
\text { Caucas-Iber. }\end{array}$ & Caucaus & Xerophyte & V-VI,VI-VII \\
\hline 35. O. caput-galli (L.) Lam. & biennial. HK & Mediter. & Ancient.Ar.d. & Xerophyte & IV.V \\
\hline 36. O. vaginalisC.A.Mey. & perennial. HK & Alban & Caucaus & Xerophyte & IV-V, V-VI \\
\hline $\begin{array}{l}\text { 37. Alhagi pseudalhagi } \\
\text { (Bieb.) Fisch. }\end{array}$ & perennial. HK & Malasiya-Turan & Ancient.Ar.d. & Xerophyte & VI- VII \\
\hline $\begin{array}{l}\text { 38. Vicia angustifolia } \\
\text { Reichard }\end{array}$ & annual. T & Europa & Boreal & Xerophyte & $\mathrm{V}, \mathrm{V}-\mathrm{VI}$ \\
\hline 39. V. cinerea Bieb. & annual. T & Turan & Desert & Xerophyte & IV-V, IV-VI \\
\hline 40. V. sativa L. & biennial. HK & Europa & Boreal & MezoXerophyte & $\mathrm{V}, \mathrm{V}-\mathrm{VI}$ \\
\hline 41.V. cordataWulf. ex Hoppe & annual. T & Unbeing known & M. o & Xerophyte & IV-V, V-VI \\
\hline
\end{tabular}




\begin{tabular}{|l|c|c|c|l|c|}
\hline 42.V. varia Host & annual. T & Europa- Mediter. & Boreal & MezoXerophyte & V-IX \\
\hline 43. Lathyrus aphaca L. & annual. T & Mediter.-Atlai & Ancient.Ar.d. & MezoXerophyte & (IV)V-VIII,VI-IX \\
\hline 44. L. annuus L. & annual. T & Mediter. & Ancient.Ar.d. & MezoXerophyte & V-VI \\
\hline 45. L. hirsutus L. & annual. T & Mediter.-Europa & Ancient.Ar.d. & MezoXerophyte & V-VI, VII-VIII \\
\hline
\end{tabular}

Analysis accoording ecological groups of cereals showed that 10 species grow in mesophyte, 50 species in xerophyte, 16 species in mesoxerophyte condition. 3 species of Legumes grow in mesophyte condition, 24 species in xerophyte, 19 species iin mesoxerophyte condition.

\section{Reference}

Agaguliev I. M. (2000). The flora and vegetation of the South-East Shirvan. Baku, Publishing House. Baku State University, 147s. (russian)

Aliev G. A., Khalilov M. U. (1976). Tugai forests of Azerbaijan (modern state and ways of improvement and recovery). B., "Elm", 135 p.(russian)

Beideman I. N. (1960). The study of plant phenology. Proc. Field geobotany, USSR Academy of Sciences, 2, 330-360. (russian)

Braun - Blanguet J. Pflanzensoziologie. (1964). Crundzuge der vegetation skunde. 3. Auflage. Wein; New York; Springer Verlage, 830 s.

Cherepanov S. K. (1995). Vascular plants of Russia and adjacent states (the former USSR). Russian edition. SPB: Peace and family, 992 p. (russian)

Flora of Azerbaijan. Azerb. SSR, Baku, 1950-1961, vol. I-VIII.(russian)

Flora of the USSR. Moscow, Leningrad, USSR Academy of Sciences, 1934-1960. m. I-XXX.(russian)

Gadzhiev V. J. (1983). Protection of the gene pool of flora and vegetation of Azerbaijan // In Proc. Protection of the natural flora of the gene pool. Novosibirsk, Nauka, 63-69.(russian)

Grossgeim A.A. (1936). Analysis of the flora of the Caucasus, Mat. BIN Az FAN SSSR, 1, 257.(russian)

Hajiyev V. D. (1990). On the protection of flora Azerbaijan // Math. Azerb. Series of biological sciences, 1.3-11. (russian)

Mammadov G. Sh., (2007). Sosial-economical and ecological bases of effective usage of soil sources in Azerbaijan. Bak1. «Elm», 856p. (azerb.)

Mammadov G. Sh., Khalilov M. Y. (2006). Ecology, environment and human. Bak1, «Elm», 608p. (azerb.)

Portenier N. N. (2000). Methodological issues highlight geographical elements of flora of the Caucasus. Bot. J., 85(6), 76-84.(russian)

Portenier N. N. (2000). The system of geographical elements of flora of the Caucasus. Bot. J., 85(9), 26-33.(russian) 
Raunkiaer C. (1934). The life forms of plants and statistical plant geography. Oxford, 48-154.

Salayev M. E. (1991). Diagnosis and classification of soils of Azerbaijan. Publishers of "Elm". Baku-240 p.(russian)

Serebryakov I. G. (1964). Life forms of higher plants and their study. In the book .: geobotany Field. - M. USSR. 3. 530 p.(russian)

Shennikov A. P. (1938). General comments on the method of routing geobotanical studies Methods of geobotanical field studies. A M: Publishing House of the USSR Academy of Sciences, 5-27. (russian)

\section{Copyright Disclaimer}

Copyright for this article is retained by the author(s), with first publication rights granted to the journal.

This is an open-access article distributed under the terms and conditions of the Creative Commons Attribution license (http://creativecommons.org/licenses/by/3.0/). 